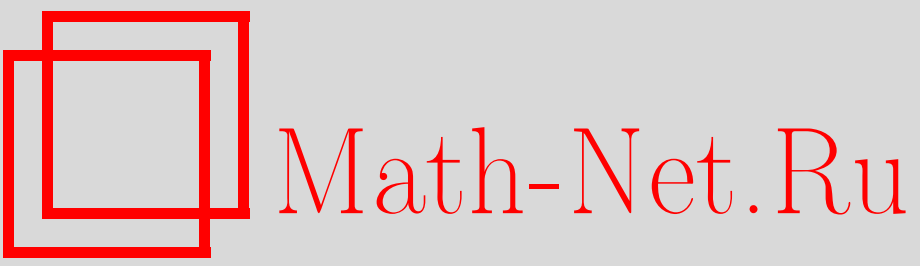

В. П. Маслов, О точно решаемой модели сверхтекучести и фазовом переходе нулевого рода (фонтанирование), ТМФ, 2004, том 141, номер 3, 411-423

DOI: https://doi.org/10.4213/tmf132

Использование Общероссийского математического портала Math-Net.Ru подразумевает, что вы прочитали и согласны с пользовательским соглашением

http://www. mathnet.ru/rus/agreement

Параметры загрузки:

IP: 3.85 .7 .115

26 апреля 2023 г., 08:40:48 
ТЕОРЕТИЧЕСКАЯ

И МАТЕМАТИЧЕСКАЯ

ФИЗИКА

Том 141, № 3

декабрь, 2004

(C) 2004 г.

В.П. Маслов*

\title{
О ТОЧНО РЕШАЕМОЙ МОДЕЛИ СВЕРХТЕКУЧЕСТИ И ФАЗОВОМ ПЕРЕХОДЕ НУЛЕВОГО РОДА (ФОНТАНИРОВАНИЕ)
}

\begin{abstract}
Приводится точно решаемая модель $N$-частичного уравнения Шредингера для симметрических состояний (бозонов), в которой определяется фазовый переход из метастабильного (сверхтекучего) состояния в нормальное, и показывается, что это фазовый переход нулевого рода со скачком свободной энергии и обращением в бесконечность теплоемкости. Показано также, что асимптотика при $N \rightarrow \infty$ решения локальных гиббсовских распределений совпадает с решением температурного уравнения Хартри, что иллюстрирует формулу автора для зависимости критерия Ландау от температуры в модели почти идеального бозе-газа Боголюбова.
\end{abstract}

Ключевые слова: сверхтекучесть, критерий Ландау, температурное уравнение Хартри, фазовый переход, температура.

До открытия сверхтекучести в физике (в термодинамике) предполагалось, что при температуре, равной нулю по Кельвину, всякое движение прекрашается ("тепловая смерть"). Это действительно следовало из старых законов термодинамики и распределения Гиббса. Когда была открыта сверхтекучесть и тот факт, что при температуре, равной нулю по Кельвину, возможно движение жидкости, возникла некоторая неувязка со старой теорией.

В работе Боголюбова 1947 года [1] на основе некоторых наводящих соображений Ландау была проинтерпретирована сверхтекучесть с помощью асимптотики при $N \rightarrow \infty$ $N$-частичного уравнения Шредингера для бозонов (симметрических по частицам собственных функций) как метастабильное состояние - уровень собственного значения более высокий, чем нижний (т.е. энергии большей, чем основное состояние наименьшей энергии). Это означало, что при малом возмушении потенциалом из достаточно широкого и естественного класса матричные элементы перехода на более низкие (меньшие) собственные значения, чем данное ("метастабильное"), малы при $N \rightarrow \infty$ (как было показано позже, экспоненциально малы). Значит, при возмушении внешним потенциалом энергия (т.е. скорость) может лишь увеличиться. Если же возникает возмушение типа “трения", это означает, что матричные элементы перехода на более высокие состояния (большие энергии) запрешены, т.е. по определению равны нулю (такое возмушение “трения", разумеется, несамосопряженно). Тем самым возмушение типа “трения" не

* Московский государственный университет, Москва, Россия 
может перевести систему из метастабильного состояния на более низкий уровень энергии ${ }^{1)}$, а значит, энергия (а в простейшем случае - скорость) останется более высокой, чем в основном (нижнем) состоянии.

1. Приведем элементарный пример на физическом уровне начала XX века полуклассики Нильса Бора. Рассмотрим одномерный случай потенциальной ямы с двумя впадинами - минимумами, один из которых (абсолютный) ниже второго (локального). Если мы рассматриваем полуклассическое приближение $h \rightarrow 0$ или если барьер между впадинами достаточно высок, то в случае, когда нет резонансов, собственные функции (а значит, и собственные значения) уравнения Шредингера, отвечаюшие собственным значениям ниже барьера, могут быть разбиты на два класса: те, носитель которых в основном лежит внутри первой впадины, и те, носитель которых в основном лежит внутри второй впадины, т.е. вне впадин значения собственных функций малы.

Предположим, что изначально частицы-бозоны расположились на уровнях энергии (собственных значениях), принадлежащих второму классу (более мелкой впадине). Под влиянием малого возмущения "трения" (если оператор возмущения не меняет носителя функции, на котором он действует; например, таким оператором является дифференциальный оператор) бозон спустится на более низкий уровень второго класса, т.е. переход на уровень первого класса почти запрешен из-за малого значения $h$ или большого барьера. В конце концов под влиянием множества возмущений “трения" все бозоны, находившиеся на уровнях второго класса, спустятся на нижний уровень второго класса "метастабильное состояние" (энергетически выгодное). Из этого состояния переход в основное состояние первого класса собственных значений почти запрешен.

Теперь перейдем к распределению Гиббса и его "ревизии”, которую, правда, в более сложной ситуации оказывается трудно объяснить привыкшим к постулату Гиббса физикам.

Все согласятся, что если барьер бесконечный и “непроницаемый”, то гиббсовскую сумму надо брать только по собственным значениям второго класса. Но очевидно, что при достаточно малом $h$ или достаточно большом барьере этот факт остается справедливым. Значит, при некотором значении параметра $h$ произойдет скачок от одной суммы Гиббса к другой? Иначе говоря, произойдет переход свободной энергии, т.е. фазовый переход нулевого рода $\left.{ }^{2}\right)$ ? На эти вопросы мы здесь отвечать не будем, поскольку это лишь наводящий пример.

Разбиение на классы в данном примере, конечно, тривиально. В примере Боголюбова оно сложнее. Однако при малых температурах, казалось бы, можно заменить впадину параболой вблизи минимума и брать сумму Гиббса по собственным значениям осциллятора. Тогда разбиения на классы не требуется, поскольку мы рассматриваем лишь собственные значения, ближайшие к нижнему уровню второго класса, и тем самым собственные значения первого класса отбрасываем. По существу, это делают Ландау в работе [2], Боголюбов [3] (т. 2, с. 218, формула (17)), а также Андреев и Мельниковский в работе [4]. Это означает, что формулу Гиббса они применяют не по всем собственным

\footnotetext{
1) То есть перевести систему в энергетически более выгодное состояние.

${ }^{2)}$ Понятие, введенное автором.
} 
значениям, как это было принято раньше, а в зависимости от метастабильного состояния, т.е. для одной и той же задачи рассматривают суммирование Гиббса по некоторым подмножествам собственных значений, т.е. локальные суммы Гиббса. Но такое приближение ("квазичастицы") формулы для фазового перехода не может дать зависимости критерия Ландау от температуры, что мы и покажем ниже на точно решаемом примеpe.

2. Рассмотрим одномерный пример с дискретным спектром так, чтобы обычное физическое определение энтропии совпало с логарифмом кратности этого собственного значения, а кратность совпала с числом ячеек. Попросту заменим одним "средним" собственным значением спектр собственных значений в заданном интервале.

Рассмотрим бозоны на окружности длины $L$. Пусть функция Гамильтона одной частицы зависит только от импульса, будем далее обозначать ее $\varepsilon(p)$. В таком случае уравнение Шредингера для одной частицы имеет вид

$$
\widehat{H} \psi(x)=\mathbf{E} \psi(x)
$$

где $\psi(x)$ - волновая функция, $x$ принимает значения на окружности, а $\widehat{H}$ - гамильтониан, дифференциальный оператор вида

$$
\widehat{H}=\varepsilon\left(-\frac{i \hbar \partial}{\partial x}\right)
$$

$\hbar$ - постоянная Планка. Полная ортонормированная система собственных функций гамильтониана (2) имеет вид

$$
\varphi_{k}(x)=\frac{e^{i p_{k} x}}{\sqrt{L}}
$$

где $p_{k}=2 \pi \hbar k / L, k$ - произвольное целое число, соответствуюшие собственные значения равны

$$
E_{k}=\varepsilon\left(p_{k}\right)
$$

В статистической физике [5] энтропия $S$ термодинамической системы как функция внутренней энергии $\mathbf{E}$ выражается формулой

$$
S(\mathbf{E})=\ln (\Gamma(\mathbf{E}-\Delta \mathbf{E}, \mathbf{E})),
$$

где $\Gamma(\mathbf{E}-\Delta \mathbf{E}, \mathbf{E})$ - число энергетических уровней с учетом кратности термодинамической системы, находящихся в интервале $(\mathbf{E}-\Delta \mathbf{E}, \mathbf{E}], \Delta \mathbf{E}-$ не зависяшая от $\mathbf{E}$ положительная величина, значение которой в термодинамическом пределе не сушественно. Перейдем от гамильтоновой функции $\varepsilon(p)$ к функции $\tilde{\varepsilon}(p)$, имеющей вид

$$
\tilde{\varepsilon}(p)=\varepsilon(n \Delta p), \quad \Delta p\left(n-\frac{1}{2}\right) \leqslant p<\Delta p\left(n+\frac{1}{2}\right),
$$

где $n$ - произвольное целое число, $\Delta p$ - некоторая положительная постоянная. Гамильтониан (2) при таком переходе изменяется соответствуюшим образом, будем обозначать 
его $\widehat{\widetilde{H}}$, собственные функции этого оператора совпадают с (3), а собственные значения (4) переходят в

$$
\widetilde{E}_{k}=\tilde{\varepsilon}\left(p_{k}\right)
$$

Будем далее считать, что постоянная $\Delta p$ имеет вид

$$
\Delta p=\frac{2 \pi \hbar G}{L}
$$

где $G$ - натуральное число. В таком случае из (6) следует, что набор энергетических уровней $(7)$ является набором $G$-кратно вырожденных энергетических уровней

$$
\lambda_{n}=\widetilde{\mathbf{E}}_{G n}=\varepsilon\left(p_{G n}\right) .
$$

Рассмотрим теперь систему из $N$ бозонов. Уравнение Шредингера для $N$ невзаимодействующих частиц имеет вид

$$
\widehat{H}_{N} \Psi\left(x_{1}, \ldots, x_{N}\right)=\mathbf{E} \Psi\left(x_{1}, \ldots, x_{N}\right)
$$

где $\Psi\left(x_{1}, \ldots, x_{N}\right)$ - симметричная функция переменных $x_{1}, \ldots, x_{N}$, а гамильтониан $\widehat{H}_{N}$ выражается формулой

$$
\widehat{H}_{N}=\sum_{j=1}^{N} \widehat{\widetilde{H}}_{j}
$$

где $\widehat{\widetilde{H}}_{j}$ - гамильтониан частищы с номером $j$, который имеет вид

$$
\widehat{\widetilde{H}}_{j}=\tilde{\varepsilon}\left(-\frac{i \hbar \partial}{\partial x_{j}}\right) .
$$

Полная ортонормированная система симметричных собственных функций гамильтониана (11) имеет вид

$$
\Psi_{\{N\}}\left(x_{1}, \ldots, x_{N}\right)=\operatorname{Symm}_{x_{1}, \ldots, x_{N}} \psi_{\{N\}}\left(x_{1}, \ldots, x_{N}\right),
$$

где $\operatorname{Symm}_{x_{1}, \ldots, x_{N}}$ - оператор симметризации по переменным $x_{1}, \ldots, x_{N},\{N\}$ - набор целых неотрицательных чисел $N_{k}, k \in \mathbb{Z}$, удовлетворяющих условию

$$
\sum_{k=-\infty}^{\infty} N_{k}=N
$$

функция $\psi_{\{N\}}\left(x_{1}, \ldots, x_{N}\right)$ равна

$$
\psi_{\{N\}}\left(x_{1}, \ldots, x_{N}\right)=\prod_{s=1}^{N} \varphi_{k_{s}}\left(x_{s}\right)
$$


а индексы $k_{1}, \ldots, k_{N}$ выражаются через набор $\{N\}$ из условий

$$
\begin{aligned}
& k_{s} \leqslant k_{s+1} \quad \text { для всех } \quad 1 \leqslant s \leqslant N-1, \\
& \sum_{s=1}^{N} \delta_{k k_{s}}=N_{k} \quad \text { для всех } k \in \mathbb{Z},
\end{aligned}
$$

$\delta_{k k^{\prime}}$ - символ Кронекера. Соответствующие собственные значения гамильтониана (11) равны

$$
\mathbf{E}(\{N\})=\sum_{k=-\infty}^{\infty} \widetilde{E}_{k} N_{k}
$$

Введем теперь взаимодействие между частицами. Будем считать, что частицы взаимодействуют парами и оператор взаимодействия частиц с номерами $j$ и $k$ есть

$$
\widehat{V}_{j k}=-\frac{V}{N} W\left(\widehat{\widetilde{H}}_{j}-\widehat{\widetilde{H}}_{k}\right)
$$

где $V>0$ - параметр величины взаимодействия, а функция $W(\xi)$ имеет вид

$$
W(\xi)= \begin{cases}1, & |\xi|<D \\ 0, & |\xi| \geqslant D\end{cases}
$$

здесь $D>0$ - параметр ширины взаимодействия по энергии. Оператор (19) соответствует взаимодействию, при котором две частицы притягиваются друг к другу и испускают квант энергии $-V / N$, если разность их энергий меньше $D$, или не взаимодействуют, если разность энергий частиц больше $D$. Гамильтониан системы $N$ частиц со взаимодействием (19) имеет вид

$$
\widehat{H}_{N}=\sum_{j=1}^{N} \widehat{\widetilde{H}}_{j}+\sum_{j=1}^{N} \sum_{k=j+1}^{N} \widehat{V}_{j k}
$$

В силу (18) суммы в выражении (20) коммутируют, поэтому набор собственных функций гамильтониана (20) совпадает с (13), также из (18) следует, что соответствующие собственные значения равны

$$
\mathbf{E}(\{N\})=\sum_{k=-\infty}^{\infty} \widetilde{E}_{k} N_{k}-\frac{V}{2 N} \sum_{k=-\infty}^{\infty} \sum_{l=-\infty}^{\infty} W\left(\widetilde{E}_{k}-\widetilde{E}_{l}\right)\left(N_{k} N_{l}-\delta_{k l} N_{k}\right) .
$$

Далее будем считать, что ширина взаимодействия достаточно мала и для нее выполняется условие

$$
D<\min _{n \neq m}\left|\lambda_{n}-\lambda_{m}\right|
$$

Набор энергетических уровней $\widetilde{E}_{k}, k \in \mathbb{Z}$, очевидно, совпадает с $G$-кратно вырожденным набором уровней (9). Окончательно получим из (22), что набор энергетических уровней (21) рассматриваемой системы $N$ частиц может быть записан в виде

$$
\mathbf{E}(\{\tilde{N}\})=\sum_{n=-\infty}^{\infty} \lambda_{n} \widetilde{N}_{n}-\frac{V}{2 N} \sum_{n=-\infty}^{\infty} \widetilde{N}_{n}\left(\widetilde{N}_{n}-1\right),
$$


где уровень $\mathbf{E}(\{\tilde{N}\})$ имеет кратность

$$
\Gamma(\{\tilde{N}\})=\prod_{n=-\infty}^{\infty} \frac{\left(G+\tilde{N}_{n}-1\right) !}{(G-1) ! \widetilde{N}_{n} !} .
$$

В формулах $(23),(24)\{\widetilde{N}\}$ обозначает набор целых неотрицательных чисел $\widetilde{N}_{n}, n \in \mathbb{Z}$, для которых выполняется условие

$$
\sum_{n=-\infty}^{\infty} \widetilde{N}_{n}=N
$$

Рассмотрим статистическую сумму для системы $N$ бозонов с гамильтонианом (20). Так как энергетические уровни и их кратности выражаются формулами (23) и (24), соответственно, то статистическая сумма при температуре $\theta$ принимает вид

$$
Z(\theta, N)=\sum_{\{\tilde{N}\}} \Gamma(\{\tilde{N}\}) e^{(-\mathbf{E}\{\tilde{N}\}) \theta}
$$

суммирование здесь берется по всем наборам $\{\tilde{N}\}$ с учетом условия (25). Учитывая (23) и (24), а также условие (25), перепишем тождественно выражение (26) в виде

$$
Z(\theta, N)=\frac{A^{N}}{2 \pi} \int_{-\pi}^{\pi} d \alpha e^{-i N \alpha} \prod_{n=-\infty}^{\infty} e^{-f_{n}\left(e^{-i \alpha} / A, \theta, N\right) / \theta}
$$

где $A \neq 0$ - произвольное число, а функции $f_{n}(z, \theta, N)$ выражаются следуюшим обра30м:

$$
f_{n}(z, \theta, N)=-\theta \ln \left(\sum_{K=0}^{N} z^{K} \frac{(G+K-1) !}{(G-1) ! K !} e^{-\lambda_{n} K / \theta+V K(K-1) /(2 N)}\right) .
$$

В дальнейшем будем считать, что $G$ зависит от $N$ так, что выполняется условие

$$
\lim _{N \rightarrow \infty} \frac{G}{N}=g>0 .
$$

Воспользуемся тождеством [6]

$$
\sum_{K=0}^{N} g(K)=\frac{g(0)+g(N)}{2}+N \sum_{l=-\infty}^{\infty} \int_{0}^{1} d x g(N x) e^{-i 2 \pi l N x}
$$

которое справедливо для любой непрерывной функции $g(x)$. Подстановка $(30)$ в $(28)$ и (27) приводит к интегралу, асимптотика которого при $N \rightarrow \infty$ в силу (29) может быть вычислена с помощью метода Лапласа. Применение метода Лапласа приводит к следующему выражению:

$$
\begin{aligned}
Z(\theta, N)= & e^{-\widetilde{F}\left(\left\{\tilde{N}^{0}\right\}, \theta\right) / \theta}\left(2 \pi \theta \sum_{l=-\infty}^{\infty} \frac{1}{\partial^{2} \widetilde{F} / \partial \widetilde{N}_{l}^{2}}\right)^{-1 / 2} \times \\
& \times \prod_{n=-\infty}^{\infty} \sqrt{\frac{2 \pi \theta}{\partial^{2} \widetilde{F} / \partial \widetilde{N}_{n}^{2}}}\left(1+O\left(\frac{1}{N}\right)\right)
\end{aligned}
$$


где $\widetilde{F}(\{\widetilde{N}\}, \theta)$ - функция вида

$$
\widetilde{F}(\{\widetilde{N}\}, \theta)=\mathbf{E}(\{\widetilde{N}\})-\theta \ln (\Gamma(\{\widetilde{N}\})),
$$

$\left\{\widetilde{N}^{0}\right\}$ - набор неотрицательных чисел $\widetilde{N}_{n}^{0}, n \in \mathbb{Z}$, для которых функция (32) минимальна при условии (25), а через $\partial^{2} \widetilde{F} / \partial \widetilde{N}_{n}^{2}$ обозначена частная производная второго порядка функции (32) по переменной $\widetilde{N}_{n}, n \in \mathbb{Z}$.

Оператор (31) имеет вид туннельного канонического оператора [6] на одномерной кривой с мерой [7]. Выражение

$$
F(\theta, N) \equiv N f(\theta, N) \equiv \widetilde{F}\left(\left\{\tilde{N}^{0}\right\}, \theta\right)
$$

является свободной энергией рассматриваемой системы $N$ бозонов при температуре $\theta$, где $f(\theta, N)$ - удельная свободная энергия. Удельная энтропия выражается через свободную энергию формулой

$$
s(\theta, N)=-\frac{\partial f}{\partial \theta}(\theta, N) .
$$

При фиксированном числе частиц $N$ формула (34) определяет кривую в двумерном фазовом пространстве с переменными $(\theta, s)$, причем удельная свободная энергия $f(\theta, N)$ является действием на этой кривой (на одномерном лагранжевом многообразии) при проецировании на переменную $\theta$, а роль постоянной Планка в каноническом операторе играет величина $1 / N$. При проецировании кривой на переменную $\theta$ могут возникнуть особенности, а на переменную $s$ это многообразие проецируется диффеоморфно, причем кривую можно задать в виде функции $\theta=\theta(s, N)$, поэтому в качестве переменной на кривой выберем удельную энтропию $s$. В этом представлении туннельньй канонический оператор имеет вид

$$
Z(s, N)=\frac{\sigma(s, N)}{\sqrt{|\partial \theta(s, N) / \partial s|}} e^{-N f(\theta(s, N), N) / \theta(s, N)},
$$

где

$$
\begin{aligned}
\sigma(s, N)= & \sqrt{\left|\frac{\partial \theta(s, N)}{\partial s}\right|}\left(2 \pi \theta(s, N) \sum_{l=-\infty}^{\infty} \frac{1}{\partial^{2} \widetilde{F} / \partial \widetilde{N}_{l}^{2}}\right)^{-1 / 2} \times \\
& \times \prod_{n=-\infty}^{\infty} \sqrt{\frac{2 \pi \theta(s, N)}{\partial^{2} \widetilde{F} / \partial \widetilde{N}_{n}^{2}}}\left(1+O\left(\frac{1}{N}\right)\right) .
\end{aligned}
$$

Выражение в правой части (35) имеет вид туннельного канонического оператора на лагранжевом многообразии $\theta=\theta(s, N)$ с мерой $\sigma(s, N) d s$.

Рассмотрим теперь вопрос о нахождении минимального значения функции (32) при условии (25). В пределе при $N \rightarrow \infty$ и при выполненном условии (29) точка минимума имеет вид

$$
\tilde{N}_{n}(\theta)=N\left(m_{n}(\theta)+o(1)\right),
$$

4 Теоретическая и математическая физика, т. 141, № 3, 2004 г. 
где $m_{n}(\theta), n \in \mathbb{Z}$, определяется из системы уравнений

$$
\lambda_{n}-V m_{n}+\theta \ln \left(\frac{m_{n}}{g+m_{n}}\right)=\mu(\theta), \quad n \in \mathbb{Z}
$$

а $\mu(\theta)$ находится из уравнения

$$
\sum_{n=-\infty}^{\infty} m_{n}=1
$$

Подстановка (37) в (32) с использованием при этом асимптотической формулы Стирлинга приводит к следуюшему равенству:

$$
\begin{aligned}
f(\theta) & \equiv \lim _{N \rightarrow \infty} f(\theta, N)=\lim _{N \rightarrow \infty} \frac{\widetilde{F}\left(\left\{\tilde{N}^{0}\right\}, \theta\right)}{N}= \\
& =\sum_{n=-\infty}^{\infty}\left(\lambda_{n} m_{n}-\frac{V}{2} m_{n}^{2}\right)+\left(\theta m_{n} \ln \left(\frac{m_{n}}{g}\right)-\theta\left(g+m_{n}\right) \ln \left(1+\frac{m_{n}}{g}\right)\right),
\end{aligned}
$$

где для краткости у функции $m_{n}(\theta)$ опушен аргумент $\theta$. Введем обозначение

$$
\omega_{n}=\lambda_{n}-V m_{n}
$$

Система уравнений (38) и (39) в обозначениях (41) принимает вид

$$
\begin{gathered}
\omega_{n}(\theta)=\lambda_{n}-V \frac{g}{e^{\left(\omega_{n}-\mu\right) / \theta}-1}, \quad n \in \mathbb{Z}, \\
\sum_{n=-\infty}^{\infty} \frac{g}{e^{\left(\omega_{n}-\mu\right) / \theta}-1}=1 .
\end{gathered}
$$

Система уравнений (42) точно совпадает с температурными уравнениями Хартри [8] для системы $N$ бозонов с гамильтонианом (20).

ЗАмЕчАниЕ. Температурные уравнения Хартри могут быть получены как асимптотика с помошью метода ультравторичного квантования термодинамики, развитого в работе [8]. Кроме того, температурные уравнения Хартри также получаются из вариационного принципа Боголюбова для свободной энергии. Таким образом, метод ультравторичного квантования и вариационный метод Боголюбова дают точный ответ для свободной энергии системы $N$ бозонов с гамильтонианом (20).

Рассмотрим решения системы уравнений (38), (39). При $\theta=0$ система имеет множество решений, которое будем нумеровать целым числом $l$ :

$$
m_{n}^{(l)}=\delta_{l n}, \quad n, l \in \mathbb{Z} .
$$

Отберем из всех номеров $l$ такие, для которых выполняется условие

$$
\nu_{n l} \equiv \lambda_{n}-\lambda_{l}+V>0 \quad \forall n \neq l .
$$


Для этих номеров сушествуют решения системы уравнений (38), (39), которые при $\theta \rightarrow 0$ сходятся к (43). Асимптотика этих решений при $\theta \rightarrow 0$ имеет вид

$$
\begin{gathered}
m_{n}^{(l)} \sim g e^{-\nu_{n l} / \theta} \quad \forall n \neq l, \\
1-m_{l}^{(l)} \sim g \sum_{n \neq l} e^{-\nu_{n l} / \theta} .
\end{gathered}
$$

Таким образом, в зависимости от спектра $\lambda_{n}, n \in \mathbb{Z}$, при достаточно малых значениях температуры $\theta$ система уравнений $(38),(39)$ имеет множество решений. Эти решения кроме точки глобального минимума содержат также точки локального минимума функции (32). Значения функции (32) в точках локального минимума равны свободной энергии метастабильных состояний. Рассмотрим функцию (32) при $\theta=0$. В этом случае она совпадает с энергетическим спектром системы бозонов (23). Рассмотрим энергию системы в случае, когда почти все частицы находятся на энергетическом уровне $\lambda_{l}$. Это означает, что вьполняются условия

$$
\tilde{N}_{n} \ll N \quad \forall n \neq l .
$$

Выражая $\widetilde{N}_{n}$ из равенства (25) и подставляя результат в (23), получим в силу (46), что энергетический спектр системы бозонов в этой области имеет вид

$$
\mathbf{E}(\tilde{N}) \approx \lambda_{l} N-\frac{V N}{2}+\sum_{n \neq l}\left(\lambda_{n}-\lambda_{l}+V\right) \tilde{N}_{n} .
$$

Теперь возможно, подобно приведенному в начале статьи примеру, выделить класс собственных значений такой, что бозоны, находящиеся на этих энергетических уровнях, под действием малых операторов "трения" спускаются в метастабильное состояние. Подробно этот процесс описан автором в работе [9] для случая двух уровней в исходном гамильтониане. Подобным же образом формулы $(32),(33),(36)$ позволяют разбить на классы собственные значения так, что каждому метастабильному состоянию (локальному минимуму функции (33)) будет отвечать свое распределение Гиббса.

Гамильтониану (20) соответствует уравнение Хартри и система уравнений в вариациях. Каждому $l \in \mathbb{Z}$ соответствует решение уравнения Хартри, описываюшее состояние

$$
\widetilde{N}_{n}^{(l)}=N \delta_{n l}
$$

Кроме того, собственные значения системы уравнений в вариациях, отвечаюшей этому решению уравнения Хартри, совпадают с

$$
\nu_{n l}=\lambda_{n}-\lambda_{l}+V, \quad n \neq l .
$$

Согласно определению, данному в [8], если собственные значения системы уравнений в вариациях для решения уравнения Хартри действительны и неотрицательны, то такое решение соответствует основному или метастабильному состоянию системы. Это означает, что всем $l$, для которых выполняется условие (44), при $\theta=0$ соответствуют метастабильные состояния системы бозонов. По аналогии со случаем нулевой температуры решениям системы уравнений (38), (39) при $\theta \neq 0$ соответствует температурное 
метастабильное состояние, если точка (37) является точкой локального минимума. Заметим теперь, что при очень больших температурах у системы уравнений $(38),(39)$ есть только одно решение, соответствуюшее глобальному минимуму функции (32). Асимптотика этого решения при $\theta \rightarrow \infty$ имеет вид

$$
n_{m}(\theta) \sim g \frac{e^{-\lambda_{m} / \theta}}{\sum_{l=-\infty}^{\infty} e^{-\lambda_{l} / \theta}} .
$$

Единственность решения при больших температурах означает, что все метастабильные состояния с увеличением температуры исчезают. Будем называть температуру, при которой исчезают метастабильные состояния, критической для этого состояния.

Исследуем поведение энтропии и теплоемкости метастабильных состояний при приближении к критической температуре. Рассмотрим метастабильное состояние, которому соответствует решение системы уравнений (38), (39), сходяшееся при $\theta \rightarrow 0$ к (43) для некоторого $l$, для которого выполняется условие (44). Будем считать далее, что функция $\varepsilon(p)$ такова, что

$$
\left.\varepsilon(p)\right|_{p=0}<\left.\varepsilon(p)\right|_{p \neq 0} .
$$

Отсюда согласно (9) следует, что

$$
\lambda_{0}<\lambda_{l} \quad \text { при } \quad l \neq 0 .
$$

Поэтому решение системы уравнений (38), (39), сходяшееся при $\theta \rightarrow 0$ к (43) для $l=0$, соответствует основному температурному состоянию системы $N$ бозонов. Кроме того, в силу (52) условие (44) становится эквивалентно условию

$$
\lambda_{l}-\lambda_{0}<V
$$

Будем считать, что $l \neq 0$ и для него выполнено (53). Условие того, что соответствующее решение $m_{n}^{(l)}(\theta)$ системы уравнений $(38),(39)$ определяет по формуле (37) точку локального минимума функции (32) при условии (25), имеет вид системы неравенств:

$$
\begin{gathered}
\alpha_{n}^{(l)}(\theta) \equiv-V+\frac{\theta g}{m_{n}^{(l)}(\theta)\left(g+m_{n}^{(l)}(\theta)\right)}>0 \quad \forall n \neq l \\
\alpha_{l}^{(l)}(\theta) \equiv-V+\frac{\theta g}{m_{l}^{(l)}(\theta)\left(g+m_{l}^{(l)}(\theta)\right)}<0, \\
-\sum_{n \neq l} \frac{\alpha_{l}^{(l)}(\theta)}{\alpha_{n}^{(l)}(\theta)}<1 .
\end{gathered}
$$

Заметим, что эти неравенства выполняются для решения (45) при $\theta \rightarrow 0$. Неравенства (54) получаются из условия положительности второй вариации функции (32), вариация берется при условии (25).

Метастабильное состояние исчезает при температуре, при которой последнее неравенство из (54) становится равенством. Будем обозначать эту критическую температуру $\theta_{\mathrm{c}}^{(l)}$. Из (54) и $(38),(39)$ при $\theta<\theta_{\mathrm{c}}^{(l)}$ следует, что $m_{n}^{(l)}(\theta)$ является растущей функцией 
переменной $\theta$ при $n \neq l$, а $m_{l}^{(l)}(\theta)$ является убываюшей функцией $\theta$. Кроме того, получается, что $m_{l}^{(l)}(\theta)>m_{n}^{(l)}(\theta)>m_{n^{\prime}}^{(l)}(\theta)$, если $\lambda_{n}<\lambda_{n^{\prime}}$ и $n, n^{\prime} \neq l$. Из указанных свойств следует, что для рассматриваемого метастабильного состояния выполняется

$$
\frac{\partial s}{\partial \theta}>0
$$

Действительно, подстановка (40) в (34) приводит к следуюшему выражению для удельной энтропии метастабильного состояния в пределе при $N \rightarrow \infty$ :

$$
s^{(l)}(\theta)=\sum_{n=-\infty}^{\infty}\left(\left(g+m_{n}^{(l)}\right) \ln \left(1+\frac{m_{n}^{(l)}}{g}\right)-m_{n}^{(l)} \ln \left(\frac{m_{n}^{(l)}}{g}\right)\right),
$$

где для краткости у функции $m_{n}^{(l)}(\theta)$ опушен аргумент $\theta$. Диффференцируя (56), получим

$$
\frac{\partial s}{\partial \theta}=\sum_{n \neq l} \frac{\partial m_{n}^{(l)}}{\partial \theta} \ln \left(\frac{g+m_{n}^{(l)}}{m_{n}^{(l)}} \frac{m_{l}^{(l)}}{g+m_{l}^{(l)}}\right) .
$$

Из последнего равенства в силу свойств $m_{n}^{(l)}(\theta)$ следует (55). Поскольку при критической температуре последнее из неравенств (54) является равенством, можно показать, что при $\theta \rightarrow \theta_{c}^{(l)}-0$ решения уравнений $(38),(39)$ ведут себя следуюшим образом:

$$
m_{n}^{(l)}(\theta)-m_{n}^{(l)}\left(\theta_{\mathrm{c}}^{(l)}\right) \approx \frac{C^{(l)}}{\alpha_{n}^{(l)}\left(\theta_{\mathrm{c}}^{(l)}\right)} \sqrt{\theta_{\mathrm{c}}^{(l)}-\theta} \quad \forall n \in \mathbb{Z},
$$

где $C^{(l)}$ - отрицательное число. Подстановка $(58)$ в (56) показывает, что производная удельной энтропии по температуре (величина, равная теплоемкости, деленной на температуру) при $\theta \rightarrow \theta_{\mathrm{c}}^{(l)}-0$ стремится к бесконечности как $1 / \sqrt{\theta_{\mathrm{c}}^{(l)}-\theta}$. Это значит, что проецирование лагранжева многообразия, соответствующего метастабильному состоянию, на ось $\theta$ становится неоднозначным в окрестности критической температуры. Производная температуры по энтропии при приближении к критической температуре обрашается в нуль, поэтому, а также в силу (57), как это уже было отмечено выше, лагранжево многообразие однозначно проецируется на ось $s$. Из обращения последнего неравенства в (54) в равенство помимо бесконечного роста теплоемкости следует, что функция $\sigma(s, N)(36)$ не имеет особенности в окрестности критической температуры. Наконец, отметим, что в силу (52) из свойств функции $m_{n}^{(l)}(\theta)$ следует, что при $\theta<\theta_{\mathrm{c}}^{(l)}$ выполняются неравенства

$$
\begin{array}{cc}
m_{0}^{(l)}(\theta)<m_{n}^{(l)}(\theta) & \forall n \neq 0, l, \\
\alpha_{0}^{(l)}(\theta)<\alpha_{n}^{(l)}(\theta) & \forall n \neq 0, l,
\end{array}
$$

которые означают, что потенциальный барьер между энергетическими уровнями $\lambda_{l}$ и $\lambda_{0}$ меньше, чем энергетический барьер между уровнями $\lambda_{l}$ и $\lambda_{n}$ при $n \neq 0, l$. Это значит, что рассматриваемая система бозонов, находяшаяся в метастабильном состоянии 
с номером $l$, при достижении критической температуры скачком изменяет свое состояние и переходит в основное температурное состояние. Это фазовый переход нулевого рода, поскольку скачком изменяются не только энтропия и теплоемкость, но также и свободная энергия.

Когда величина $\min _{n \neq 0}\left|\lambda_{n}-\lambda_{0}\right|=\delta$ мала, разность свободных энергий при фазовом переходе нулевого рода из самого нижнего метастабильного состояния в основное состояние также является малой, а теплоемкость при таком переходе имеет особенность. Асимптотика статистической суммы около критической точки дается асимптотикой канонического оператора в окрестности фокальной точки. Эта асимптотика имеет вид функции типа Эйри от мнимого аргумента и имеет особенность при $N \rightarrow \infty$. Можно подобрать параметры $\delta \ll 1$ и $N \gg 1$ так, что вид точки фазового перехода будет в точности совпадать с графиком для $\lambda$-точки [10].

В заключение для сравнения рассмотрим статистическую сумму метастабильного состояния только как сумму по квазичастицам. Это означает, что точный спектр (23) энергий системы $N$ бозонов с гамильтонианом (20) заменим на асимптотику этого спектра около метастабильного состояния (48), для которого выполнено условие (44):

$$
\widetilde{\mathbf{E}}^{(l)}(\tilde{N})=\lambda_{l} N-\frac{V N}{2}+\sum_{n \neq l}\left(\lambda_{n}-\lambda_{l}+V\right) \widetilde{N}_{n}
$$

Статистическая сумма в этом случае выражается формулой, аналогичной (26):

$$
\widetilde{Z}^{(l)}(\theta, N)=\sum_{\{\tilde{N}\}} \Gamma(\{\tilde{N}\}) e^{-\widetilde{\mathbf{E}}^{(l)}(\{\tilde{N}\}) / \theta} .
$$

Так же как и для статистической функции (26), асимптотика суммы (61) в пределе при $N \rightarrow \infty$ определяется минимумом функции

$$
\widetilde{F}^{(l)}(\{\tilde{N}\}, \theta)=\widetilde{\mathbf{E}}^{(l)}(\{\tilde{N}\})-\theta \ln (\Gamma(\{\tilde{N}\})) .
$$

Спектр (60) является спектром идеального бозе-газа, поэтому статистическая сумма (61) не имеет особенностей ни при каких значениях температуры. Кроме того, функция (62) имеет единственную точку минимума, который является глобальным и не исчезает ни при какой температуре. Действительно, температурные уравнения Хартри, решения которых определяют минимум функции (62) в пределе при $N \rightarrow \infty$, в случае спектра (60) принимают следующий тривиальный вид:

$$
\begin{gathered}
\omega_{n}^{(l)}=\nu_{n l}=\lambda_{n}-\lambda_{l}+V \quad \forall n \neq l, \\
\omega_{l}=0 .
\end{gathered}
$$

Таким образом, аналог системы уравнений (38) для спектра (60) при любом значении температуры имеет единственное решение вида

$$
m_{n}^{(l)}(\theta)=\frac{g}{e^{\left(\omega_{n}^{(l)}-\mu\right) / \theta}-1}
$$


где $\mu$ однозначно определяется. Асимптотика решения (64) при $\theta \rightarrow 0$ совпадает с (45), однако при не малых температурах решение (64) не совпадает с решением уравнений (38), (39), самое важное отличие состоит в том, что для решения (64) не сушествует критической температуры, при которой это решение исчезает или приводит к особенности теплоемкости. Таким образом, считать статистическую сумму метастабильного состояния по квазичастицам можно только при малых температурах. При температуpax, близких к температуре фазового перехода, расчет приводит к принципиально другим результатам.

Все сказанное выше справедливо для произвольного вида функции $\varepsilon(p)$. В частности, можно в качестве $\varepsilon(p)$ выбрать как фононно-ротонную модель [1], [11], так и старую модель Ландау [2].

Отметим в заключение, что из формулы (17) из работы Боголюбова [3] следует скачок второй производной (как бы “фазовый переход второго рода"). Однако Боголюбов, насколько мне известно, понимал, что ответ не будет совпадать с истинным фазовым переходом, который, как показано в данной работе, на самом деле будет фазовым переходом нулевого рода.

Фазовый переход нулевого рода экспериментально, по сушеству, был открыт в 1938 году Дж. Алленом и Х. Джонсом для жидкого ${ }^{2}$ Не (см. [12]). В капилляре диаметром $10^{-4}$ см "протекал" сверхтекучий гелий. В некоторой точке, над которой была проделана крохотная дырка, он нагревался светом до точки фазового перехода, после чего уже нормальная жидкость из-за вязкости не могла протиснуться в узкий капилляр и через дырочку начинал бить фонтан высотой в 14 см. С точки зрения динамики этот эффект легко объясним. Но сверхтекучесть - не течение, а состояние, как, например, лед, и описывается не динамикой, а термодинамикой. И переход из одного термодинамического состояния в другое происходит со скачком свободной энергии, т.е. все частицы из состояния, которое было метастабильным, каскадом ринутся в основное состояние, когда барьер перестанет существовать.

\section{Список литературы}

[1] Н. Н. Боголюбов. Изв. АН СССР. Сер. физич. 1947. Т. 11. № 1. С. 77.

[2] Л. Д. Ландау. ЖЭТФ. 1941. Т. 11. С. 592.

[3] Н. Н. Боголюбов. Избранные труды в трех томах. Т. 1, 2. Киев: Наукова думка, 1970.

[4] А. Ф. Андреев, Л. А. Мельниковский. Письма в ЖЭТФ. 2003. Т. 78. № 9. С. 1063.

[5] Л. Д. Ландау, Е. М. Лифииц. Статистическая физика. Ч. 1. М.: Наука, 1976.

[6] В. П. Маслов. Операторные методы. М.: Наука, 1973.

[7] В. П. Маслов. Асимптотические методы и теория возмущений. М.: Наука, 1988.

[8] В. П. Маслов. Квантование термодинамики и ультравторичное квантование. М.: Институт компьютерных исследований, 2001.

[9] В. П. Маслов. Функц. анализ и его прилож. 2003. Т. 37. № 2. С. 16.

[10] И. А. Квасников. Термодинамика и статистическая физика. Т. 1. Теория равновесных систем: Термодинамика. М.: Эдиториал УРСС, 2002.

[11] L. D. Landau. J. Phys. USSR. 1947. V. 11. P. 91.

[12] И. А. Квасников. Термодинамика и статистическая физика. Т. 2. Теория равновесных систем: Статистическая физика. М.: Эдиториал УРСС, 2002.

Поступила в редакцию 21.VI.2004 г. 